\title{
Measurement of cell microrheology by magnetic twisting cytometry with frequency domain demodulation
}

\author{
MARINA PUIG-DE-MORALES, ${ }^{1}$ MIREIA GRABULOSA, ${ }^{1}$ JORDI ALCARAZ, ${ }^{1}$ \\ JOAQUIM MULLOL, ${ }^{2}$ GEOFFREY N. MAKSYM, ${ }^{3}$ \\ JEFFREY J. FREDBERG, ${ }^{4}$ AND DANIEL NAVAJAS ${ }^{1}$ \\ ${ }^{1}$ Unitat Biofísica i Bioenginyeria, Facultat Medicina, Universitat Barcelona-IDIBAPS and ${ }^{2}$ Hospital \\ Clínic, 08036 Barcelona, Spain; ${ }^{3}$ School of Biomedical Engineering, Dalhousie University, Halifax, \\ Nova Scotia, Canada B3H 3J5; and ${ }^{4}$ Harvard School of Public Health, Boston, Massachusetts 02115
}

Received 14 February 2001; accepted in final form 23 April 2001

\begin{abstract}
Puig-De-Morales, Marina, Mireia Grabulosa, Jordi Alcaraz, Joaquim Mullol, Geoffrey N. Maksym, Jeffrey J. Fredberg, and Daniel Navajas. Measurement of cell microrheology by magnetic twisting cytometry with frequency domain demodulation. J Appl Physiol 91: 1152-1159, 2001.Magnetic twisting cytometry (MTC) (Wang N, Butler JP, and Ingber DE, Science 260: 1124-1127, 1993) is a useful technique for probing cell micromechanics. The technique is based on twisting ligand-coated magnetic microbeads bound to membrane receptors and measuring the resulting bead rotation with a magnetometer. Owing to the low signal-to-noise ratio, however, the magnetic signal must be modulated, which is accomplished by spinning the sample at $\sim 10 \mathrm{~Hz}$. Present demodulation approaches limit the MTC range to frequencies $<0.5 \mathrm{~Hz}$. We propose a novel demodulation algorithm to expand the frequency range of MTC measurements to higher frequencies. The algorithm is based on coherent demodulation in the frequency domain, and its frequency range is limited only by the dynamic response of the magnetometer. Using the new algorithm, we measured the complex modulus of elasticity (G*) of cultured human bronchial epithelial cells (BEAS-2B) from 0.03 to $16 \mathrm{~Hz}$. Cells were cultured in supplemented RPMI medium, and ferromagnetic beads $(\sim 5 \mu \mathrm{m})$ coated with an RGD peptide were bound to the cell membrane. Both the storage $\left(G^{\prime}\right.$, real part of $\left.G^{*}\right)$ and loss $\left(G^{\prime \prime}\right.$, imaginary part of $\left.G^{*}\right)$ moduli increased with frequency as $\omega^{\alpha}(2 \pi \times$ frequency) with $\alpha \approx 1 / 4$. The ratio $\mathrm{G}^{\prime \prime} / \mathrm{G}^{\prime}$ was $\sim 0.5$ and varied little with frequency. Thus the cells exhibited a predominantly elastic behavior with a weak power law of frequency and a nearly constant proportion of elastic vs. frictional stresses, implying that the mechanical behavior conformed to the so-called structural damping (or constant-phase) law (Maksym GN, Fabry B, Butler JP, Navajas D, Tschumperlin DJ, LaPorte JD, and Fredberg JJ, J Appl Physiol 89: 1619-1632, 2000). We conclude that frequency domain demodulation dramatically increases the frequency range that can be probed with MTC and reveals that the mechanics of these cells conforms to constant-phase behavior over a range of frequencies approaching three decades.
\end{abstract}

cell mechanics; cell viscoelasticity; complex elastic modulus; power law rheology; structural damping; magnetic tweezers

MECHANICAL PROPERTIES OF THE CELL play an important role in essential cellular functions such as mechano-

Address for reprint requests and other correspondence: D. Navajas, Unitat Biofísica i Bioenginyeria, Facultat Medicina, Casanova 143, 08036-Barcelona, Spain (E-mail: dnavajas@medicina.ub.es). transduction, shape stability, motility, apoptosis and DNA synthesis (12-14, 19, 23). Techniques for studying cell mechanics include cell poking (6), atomic force microscopy (18), optical tweezers (28), laser tracking microrheology (27), magnetic bead microrheometry (2), and magnetic twisting cytometry (MTC) (23). MTC, in particular, has proved to be a useful tool for exploring force transmission across the cell membrane and for assessing cell stiffness and its changes (11, 15, 23, 25, 26). This technique was first introduced by Crick (4) and Crick and Hughes (5) and was further refined by Valberg (21), Wang et al. in 1993 (23), and, most recently, by Maksym et al. (16). As employed most frequently, an external magnetic field is used to apply a twisting stress on ligand-coated magnetic microbeads $(\sim 5 \mu \mathrm{m})$ bound to membrane receptors. As the bead rotates, mechanical stresses opposing that rotation are developed within the cell to which the bead is attached. As such, mechanical properties of the cell can be derived from measurements of the applied rotatory torque and the resulting bead rotation recorded from an in-line magnetometer.

To separate signal from noise, the magnetic field generated by the beads is modulated by spinning the sample at $\sim 10 \mathrm{~Hz}$. Demodulation is performed in the time domain by multiplying the recording with a reference signal phase locked with the spinning rate and by low-pass filtering the product with a cutoff frequency of $\sim 0.5 \mathrm{~Hz}$ (22). By using time domain demodulation, the behavior of a variety of cells and multiple interventions has been studied using either step unidirectional twisting fields $(11,15,23,25,26)$ or oscillatory twisting fields (16). In the latter case, the dynamic behavior of cultured airway smooth muscle cells has been measured from 0.05 to $0.4 \mathrm{~Hz}$. However, time domain demodulation restricts the range of oscillatory measurements to frequencies up to one decade below the spinning frequency. This demodulation approach represents an important limitation of MTC to probe cell microrheology over a wider frequency range.

The costs of publication of this article were defrayed in part by the payment of page charges. The article must therefore be hereby marked "advertisement" in accordance with 18 U.S.C. Section 1734 solely to indicate this fact. 
The aims of this report were to implement a novel frequency domain algorithm to expand the frequency range of MTC oscillatory measurements to higher frequencies and to use the new algorithm to study oscillation mechanics of adherent cells over an extended frequency range. The new algorithm is based on coherent demodulation in the frequency domain, as opposed to time domain demodulation as used previously (16, $23)$. The signal is recovered from the spectral values located at the harmonics of the oscillatory frequency. The performance of the algorithm was assessed with a realistic simulation of MTC recordings with a viscoelastic model. The new demodulation approach was then applied to measure the complex modulus of elasticity of cultured human airway epithelial cells from 0.03 to $16 \mathrm{~Hz}$.

\section{METHODS}

\section{MTC Demodulation}

Figure 1 depicts the basis of MTC measurements. Ferromagnetic microbeads $(\sim 5 \mu \mathrm{m}$ in diameter) coated with a specific ligand to a selected receptor are attached to the cell membrane. The microbeads are magnetized in the $\phi_{\mathrm{o}}$ direction with a brief large magnetic pulse $(\sim 1 \mathrm{~ms}, \sim 100 \mathrm{mT})$. In conventional MTC step experiments, the beads are magnetized in the horizontal plane $\left(\phi_{\mathrm{o}}=0\right)$. However, in oscillatory experiments it is more convenient to magnetize the beads at $\phi_{\mathrm{o}} \sim \pi / 4$ to avoid signal rectification around $\phi=0$ and to improve magnetic sensitivity of measurements (16). The horizontal component of the magnetic field induced by the remanent magnetic moment created in the beads is measured in the $y$-axis $[B(t)]$ with a magnetometer. A rotatory torque is applied to the beads with a weak $(<3 \mathrm{mT})$ vertical magnetic field strength $[H(t)]$. The specific torque $[T(t)]$ is the torque per unit of bead volume divided by a geometric scaling factor (6 for a sphere) and is defined as

$$
T(t)=c_{b} H(t) \cos \phi(t)
$$

where $\phi(t)$ is the angle between the remanent magnetic moment and the horizontal plane and $c_{b}$ is the bead calibration constant (24). When the sample is subjected to sinusoi-

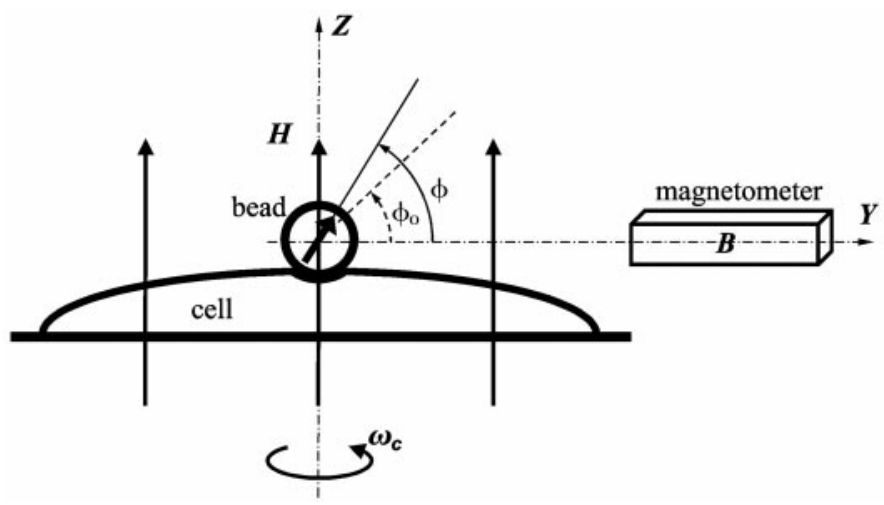

Fig. 1. Diagram of the magnetic twisting cytometry (MTC) measurements. Ferromagnetic microbeads attached to the cell membrane are magnetized in the $\phi_{\mathrm{o}}$ direction. The bead is twisted $(\phi)$ by applying a vertical magnetic field $(H)$. The bead rotation is computed from the change in the magnetic field recorded with a magnetometer $(B)$. The magnetic signal from the bead is modulated by spinning the sample $\left(\omega_{\mathrm{c}}\right)$. dal twisting field with amplitude $H_{a}$ and angular frequency $\omega_{\mathrm{o}}(\omega=2 \pi f, f$ is frequency $)$

$$
H(t)=H_{a} \sin \omega_{0} t
$$

the rotation induced in the beads is a periodic function that can be represented by the Fourier series (17)

$$
\phi(t)=A_{\mathrm{o}}+\sum A_{k} \sin \left(k \omega_{0} t+\Phi_{k}\right), \quad k=1,2,3, \ldots
$$

where $A_{\mathrm{o}}$ is the mean value of $\phi(t)$, and $A_{k}$ and $\phi_{k}$ are the magnitude and phase, respectively, of the oscillatory frequency $(k=1)$ and its harmonics $(k \geq 2)$.

We characterize the oscillatory response of the cell by the complex modulus of elasticity $\left[\mathrm{G}^{*}(\omega)\right]$ defined as the complex ratio in the Fourier domain between the applied specific torque and the induced bead rotation computed at the oscillatory frequency

$$
\mathrm{G}^{*}(\omega)=T(\omega) / \phi(\omega)
$$

Its magnitude, $G(\omega)$, is a measure of the resistance of the cell to deformation and its phase angle, $\delta(\omega)$, is an index of solidlike $(\delta=0)$ or liquidlike $(\delta=\pi / 2)$ behavior. Alternatively, $\mathrm{G}^{*}(\omega)$ can be separated into real and imaginary components as

$$
\mathrm{G}^{*}(\omega)=\mathrm{G}^{\prime}(\omega)+j \mathrm{G}^{\prime \prime}(\omega)
$$

where $j=\sqrt{-1} \cdot \mathrm{G}^{\prime}(\omega)$ and $\mathrm{G}^{\prime \prime}(\omega)$ are the storage and loss moduli, respectively. The ratio $\mathrm{G}^{\prime \prime}(\omega) / \mathrm{G}^{\prime}(\omega)=\tan \delta(\omega)$ is known as the loss tangent or equivalently as the hysteresivity $[\eta(\omega)](8)$ and reflects the relative proportion of dissipated and stored mechanical energy per cycle of sinusoidal deformation.

Measuring $\mathrm{G}^{*}(\omega)$ requires the computation of $\phi(t)$ from the magnetometer recording. The magnetic signal generated in the magnetometer by the beads is

$$
B(t)=B_{\mathrm{o}} \rho(t) \cos \phi(t)
$$

where $B_{\mathrm{o}}$ is the magnetic field measured just after magnetization at $\phi=0$ and $\rho(t)$ is the relaxation function accounting for the slow magnetic decay due to random rotation of the beads that can be approximated to an exponential decay function $\mathrm{e}^{-\gamma t}(22)$. The actual magnetometer recording, however, has additional correlated and random noise components

$$
B(t)=B_{\mathrm{o}} \rho(t) \cos \phi(t)+B_{\mathrm{s}} \sin \omega_{0} t+n(t)
$$

where $B_{\mathrm{s}}$ is the amplitude of the correlated spill field induced by the twisting solenoid in the magnetic probes due to small misalignment and $n(t)$ is the random noise of the system.

The conventional method of separating signal from correlated and random noise is to modulate the magnetic field by spinning the sample with angular frequency $\omega_{c}(22,23)$. In this case, the magnetometer recording is

$$
B(t)=B_{\mathrm{o}} \rho(t) c(t) \cos \phi(t)+B_{\mathrm{s}} \sin \omega_{\mathrm{o}} t+n(t)
$$

with the modulation carrier $[c(t)]$ of the form

$$
c(t)=\sin \left[\omega_{c} t+\theta_{c}(t)\right]
$$

where $\theta_{c}(t)$ accounts for small spinning instability of the system.

To demodulate the recording, a sinusoidal reference $[r(t)]$ phase locked with the carrier is digitally synthesized from synchronization pulses obtained with a photodiode attached to the spinning system. Multiplying the recording $(E q .8)$ by $r(t)$, assuming that $r(t)=c(t)$, and using the trigonometric identity $\sin ^{2} A=1 / 2(1-\cos 2 A)$, it follows that 


$$
\begin{array}{r}
B(t) r(t)=1 / 2 B_{\mathrm{o}} \rho(t) \cos \phi(t)-1 / 2 B_{\mathrm{o}} \rho(t) \cos \phi(t) \\
\times \cos \left\{2\left[\omega_{c} t+\theta_{c}(t)\right]\right\}+B_{\mathrm{s}} \sin \omega_{\mathrm{o}} t \sin \left[\omega_{c} t+\theta_{c}(t)\right] \\
+n(t) \sin \left[\omega_{c} t+\theta_{c}(t)\right]
\end{array}
$$

Dividing by $B_{\mathrm{o}} \rho(t) / 2$ and rearranging the terms we have

$$
\begin{gathered}
x(t)=2 B(t) r(t) /\left[B_{\mathrm{o}} \rho(t)\right]=\cos \phi(t)-\cos \phi(t) \\
\times \cos \left\{2\left[\omega_{c} t+\theta_{c}(t)\right]\right\}+\left\{2 /\left[\mathrm{B}_{\mathrm{o}} \rho(t)\right] B_{\mathrm{s}} \sin \omega_{\mathrm{o}} t \sin \left[\omega_{c} t+\theta_{c}(t)\right]\right\} \quad(11) \\
+\left\{2 /\left[B_{\mathrm{o}} \rho(t)\right] n(t) \sin \left[\omega_{c} t+\theta_{c}(t)\right]\right\}
\end{gathered}
$$

If $\cos \phi(t)$ has only low-frequency components, it can be recovered by low-pass filtering $x(t)$ in the time domain $\left[x_{\mathrm{LP}}(t)\right]$ with a cutoff frequency far below $\omega_{c}(22)$. Thus the desired signal, $\phi(t)$, is computed as $\cos ^{-1}\left[x_{L P}(t)\right]$. Using a low cutoff frequency improves the signal-to-noise ratio (SNR) but limits the frequency band of the recovered signal. Typically, a cutoff frequency of $\sim 0.5 \mathrm{~Hz}$ is used, which limits MTC measurements to this low-frequency range.

Frequency domain demodulation algorithm. An alternative approach to expanding the frequency range of MTC oscillatory experiments is to compute the spectrum of $x(t)$ and recover $\phi(t)$ from the spectral peaks located at $k \omega_{\mathrm{o}}(k=0,1$, $2, \ldots$. . With this approach, the frequency band of the recovered signal is limited only by the sampling frequency $\left(f_{\mathrm{s}}\right)$ of the recording. In particular, oscillation frequency can be higher than spinning frequency. On the basis of this approach, we implemented the following frequency domain demodulation algorithm to measure oscillation mechanics of the cell with MTC.

First, the algorithm removes the mean value from the recording and corrects it for the low-pass frequency response of the magnetometer. Second, $x(t)$ is computed (Eq. 11) by multiplying the corrected recording by the corresponding reference and dividing the result by $B_{\circ} \rho(t) / 2$. Third, the spectrum $[X(\omega)]$ of a segment of $x(t)$ including a whole number of oscillatory periods $\left(T_{\mathrm{o}}=1 / \omega_{\mathrm{o}}\right)$ of the twisting field oscillation is computed by fast Fourier transform (FFT). Fourth, $\phi(t)$ is recovered from the first harmonics as

$$
\begin{aligned}
\phi(t)=\cos ^{-1}\left\{\mathrm{FFT}^{-1}\left[X(\omega) \sum \delta\left(\omega-k \omega_{0}\right)\right]\right\} & \\
k & =-4,-3, \ldots, 4
\end{aligned}
$$

where $\delta(\omega)$ is the Dirac delta function. Finally, G*( $\left.\mathrm{G}^{*}\right)$ is computed as FFT $[T(t)] / \operatorname{FFT}[\phi(t)]$ at $\omega=\omega_{\mathrm{o}}(E q$. 4). To estimate the reliability of the spectral components used to recover the signal, $x(t)$ is divided into four consecutive fragments, and their spectra at $k \omega_{\mathrm{o}}(k=0,1, \ldots, 4)$ are computed. Only the harmonics of the oscillatory frequency with variability lower than a predefined threshold are used in $E q .12$ to recover the signal. For a more efficient computation, the oscillation frequency is adapted to the duration of each experiment and to the sampling frequency to have $x(t)$ with a power of 2 points and to have a whole number of oscillatory periods in $x(t) / 4$.

\section{Simulation Study}

The mechanical response of the cell for sinusoidal twisting was simulated by means of a viscoelastic mathematical model with constant $\mathrm{G}^{\prime}=50 \mathrm{~Pa}$ and $\mathrm{G}^{\prime \prime}=15 \mathrm{~Pa}$. The rotation induced in the bead by the twisting field was obtained by solving (Matlab, The MathWorks, Natick, MA) the differential equation of the model for $\phi(t)$

$$
c_{b} H(t) \cos \phi(t)-\mathrm{G}^{\prime \prime} \dot{\phi}(t) / \omega_{0}-\mathrm{G}^{\prime}\left[\phi(t)-\phi_{0}\right]=0
$$

where the overdot denotes differentiation with respect to time. This model has constant modulus amplitude $G=52.2$ $\mathrm{Pa}$ and phase angle $\delta=6.7^{\circ}(\tan \delta=0.3)$. The simulations were carried out from 0.03 to $16 \mathrm{~Hz}$ with $c_{b}=3 \mathrm{~Pa} / \mathrm{mT}$ and twisting field of $2 \mathrm{mT}$ amplitude. This twisting field corresponds to a specific torque amplitude of $\sim 6 \mathrm{~Pa}$ that produces an amplitude oscillation of $\sim 5^{\circ}$. The oscillation frequencies were adjusted to obtain a whole number of periods in 131.072 s $\left(2^{15}\right.$ points $\left./ f_{\mathrm{s}}\right)$.

Sample recordings of carrier, $c(t)$, and reference signals, $r(t)$, were obtained with a sample of magnetic powder glued in epoxy, which produced a strong magnetic signal $(\sim 0.6 \mu \mathrm{T})$. This sample was spun without applying the twisting field. Because random noise was negligible for the high magnetic field of this sample, we took eight recordings as representative of carrier and reference signals. The random noise of the system, $n(t)$, was characterized from eight recordings taken without magnetic sample or twisting field. The noise recordings were low-pass filtered (Butterworth analog filter, 500 $\mathrm{Hz}, 8$ poles) to eliminate the $5-\mathrm{kHz}$ noise of the magnetometer (Förster Magnetoscop 1068). All the recordings had a duration of $131.072 \mathrm{~s}$ and were digitized at $250 \mathrm{~Hz}$.

Simulated MTC recordings for the different oscillatory frequencies were obtained as follows. First, the rotation induced in the bead, $\phi(t)$, by sinusoidal twisting of $2 \mathrm{mT}$ amplitude was computed for the different oscillatory frequencies from $E q$. 13. Second, the magnetic signal from the beads, $B(t)$, was computed as Eq. 6 with $B_{\mathrm{o}}=1.41 \mathrm{nT}$ and $\gamma=10^{-3}$ $\mathrm{s}^{-1}$. Third, this signal was multiplied by a carrier recording. Finally, a spill field of $0.5 \mathrm{nT}$ amplitude and a random noise recording were added ( $E q .8)$. Eight simulated MTC recordings at each oscillatory frequency were demodulated by using the reference signal corresponding to each carrier recording. Harmonics of the fundamental frequency with variability higher than $10 \%$ were rejected. Finally, $G^{*}(\omega)$ was computed, and its magnitude and phase angle were compared with the actual model values. The simulation was also carried out without adding random noise.

\section{Cellular Study}

Reagents. RPMI 1640 culture medium and fetal calf serum were purchased from Biological Industries (Kibbutz Beit Haemek, Israel). Insulin, hydrocortisone, human transferrin, $\mathrm{Na}_{2} \mathrm{SeO}_{3}$, and BSA were obtained from Sigma Chemical (St. Louis, MO); penicillin-streptomycin solution and HEPES buffer from GIBCO (Gaithersburg, MD); epidermal growth factor from Calbiochem (La Jolla, CA); type I rat tail collagen from Upstate Biotechnology (Lake Placid, NY); L-glutamine from ICN Pharmaceuticals (Costa Mesa, CA); amphotericin B from Squibb (Esplugues de Llobregat, Spain). Trypsin solution and soybean trypsin inhibitor were purchased from Biofluids (Rockville, MD). Synthetic RGD (Arg-Gly-Asp)-containing peptide (Peptide 2000) was purchased from Telios (San Diego, CA).

Cell culture. The study was carried out in BEAS-2B cells, a human bronchial epithelial cell line transformed by the adenovirus $12-S V 40$ hybrid. The BEAS-2B cell line was a gift from J. E. Lechner, National Cancer Institute, National Institutes of Health (Bethesda, MD). The cells were cultured in $150-\mathrm{cm}^{2}$ plastic flasks coated with type I rat tail collagen and maintained in RPMI culture medium supplemented with $1 \%$ fetal calf serum, penicillin (100 U/ml), streptomycin (100 $\mu \mathrm{g} / \mathrm{ml})$, amphotericin B $(2 \mu \mathrm{g} / \mathrm{ml})$, insulin $(1.74 \mu \mathrm{g} / \mathrm{ml})$, hydrocortisone $(2.75 \mu \mathrm{g} / \mathrm{ml})$, human transferrin $(10 \mu \mathrm{g} / \mathrm{ml})$, $\mathrm{Na}_{2} \mathrm{SeO}_{3}(50 \mathrm{nM})$, L-glutamine $(1 \mathrm{mM})$, epidermal growth factor $(10 \mathrm{ng} / \mathrm{ml})$, and HEPES buffer $(10 \mathrm{mM})$. The cells were 
incubated at $37^{\circ} \mathrm{C}$ in a $95 \%$ air- $5 \% \mathrm{CO}_{2}$ environment with $100 \%$ humidity. The medium was replaced every 2 days. After reaching $\sim 80 \%$ subconfluence, the cells were detached from the culture flasks with $0.02 \%$ trypsin, $1 \%$ polyvinyl pyrrolidine, and $0.02 \%$ ethylene glycol bis, and then soybean trypsin inhibitor was added. Dissociated cells were centrifuged, washed, counted, and resuspended in supplemented RPMI medium.

The day before the experiments were performed, cells were serum deprived and supplemented with 1\% BSA. After reaching confluence, the cells were plated $\left(3 \times 10^{4}\right.$ cells/well $)$ on bacteriological plastic wells $(6.4 \mathrm{~mm}, 96$-well Removawell, Immulon II, Dynatech, Chantilly, VA) that were coated overnight with type I rat tail collagen (500 ng/well). After 6 h, 30 $\mu \mathrm{g}$ of RGD-coated ferromagnetic beads $\left(\sim 5 \mu \mathrm{m}\right.$ diameter, $c_{b}$ $=3.1 \mathrm{~Pa} / \mathrm{mT}$ ) produced at the Harvard School of Public Health were added to each well. The beads were coated with the RGD peptide in carbonate buffer ( $\mathrm{pH}$ 9.4) $(50 \mu \mathrm{g}$ peptide $\cdot \mathrm{mg} \mathrm{bead}^{-1} \cdot \mathrm{ml}$ carbonate buffer $^{-1}$ ) and stored overnight at $4^{\circ} \mathrm{C}$ to facilitate protein absorption onto the beads. After 10-15 min incubation, unbound beads were washed twice with serum-deprived medium supplemented with $1 \%$ $\mathrm{BSA}$, and the oscillatory measurements were performed.

MTC measurements. The cellular samples $(n=9)$ were placed in the MTC device and maintained at $37^{\circ} \mathrm{C}$. The beads were magnetized, and $20 \mathrm{~s}$ afterward they were subjected to an oscillatory twisting field of 2-mT amplitude. Oscillation frequencies ranging from $\sim 0.03$ to $\sim 16 \mathrm{~Hz}$ were applied in random order. The frequencies were adjusted to obtain a whole number of periods in $131.072 / 4 \mathrm{~s}$. The total duration of the oscillation was $131.072 \mathrm{~s}$ plus an additional cycle. After the oscillation, $20 \mathrm{~s}$ without twisting field was also recorded. The recordings were low-pass filtered (Butterworth analog filter, $500 \mathrm{~Hz}, 8$ poles), digitized at $250 \mathrm{~Hz}$, and digitally corrected for the low-pass frequency response of the magnetometer (cutoff $200 \mathrm{~Hz}$ ). The recordings were first time domain demodulated, and $B_{\mathrm{o}}$ and $\gamma$ were determined by fitting the exponential function $B_{\mathrm{o}} \mathrm{e}^{-\gamma t}$ to the 20 -s segments recorded before and after the oscillation. The first cycle of oscillation was discarded to avoid transients, and frequency domain demodulation was carried out with the remaining $131.072 \mathrm{~s}$ of oscillation.

\section{Modeling}

$\mathrm{G}^{*}(\omega)$ data were fit with the power law constant-phase model $(9,10)$

$$
\mathrm{G}^{*}(\omega)=\mathrm{G}_{\mathrm{s}}^{\prime}(1+j \eta)\left(\omega / \omega_{\mathrm{s}}\right)^{\alpha}
$$

where

$$
\eta=\tan (\pi \alpha / 2)
$$

and $\omega_{\mathrm{s}}$ is a scale factor for frequency, which, for simplicity, we take as $\omega_{\mathrm{s}}=1 \mathrm{~s}^{-1}$. This model assumes that both $\mathrm{G}^{\prime}(\omega)$ and $\mathrm{G}^{\prime \prime}(\omega)$ follow a power law with the same exponent. Consequently, the magnitude of the complex modulus also follows a power law, with the same exponent $\mathrm{G}^{*}=\left(1+\eta^{2}\right)^{1 / 2} \mathrm{G}_{\mathrm{S}}^{\prime}$ $\left(\omega / \omega_{\mathrm{s}}\right)^{\alpha}$. In addition, the phase angle does not depend on frequency and is related to the power law exponent as $\delta=$ $\pi \alpha / 2$.

\section{Statistical Analysis}

Dependence of the relative error in magnitude and of the absolute error in phase angle on the oscillation frequency was tested in the simulation study with linear regression analysis. In the cellular study, the frequency dependence of the coefficient of variation $(\mathrm{CV}=100 \times \mathrm{SD} / \mathrm{mean})$ of $\mathrm{G}^{\prime}$ and $\mathrm{G}^{\prime \prime}$ was tested with linear regression analysis. The power law constant-phase model was fitted to the cellular data by nonlinear regression analysis (SigmaPlot, SPSS, Chicago, IL). Statistical tests were taken as significant when $P<0.05$.

\section{RESULTS}

The average root mean square value ( $\mathrm{rms}$ ) of the random noise computed with the eight sample records was $0.17 \mathrm{nT}$. The spectrum of the noise decreased markedly with increasing frequency according to PSD $\left(\mathrm{nT}^{2} / \mathrm{Hz}\right)=1.44 \times 10^{-4}+2.55 \times 10^{-4} / f\left(f\right.$ in $\mathrm{Hz} ; r^{2}=$ 0.912 ), where PSD is the one-sided power spectral density.

The algorithm estimated the complex modulus of the model very well in the simulation without noise (Fig. 2 ). At all frequencies, the mean errors were $<0.2 \%$ $(\mathrm{SD}<0.5 \%)$ in magnitude and $<0.2^{\circ}\left(\mathrm{SD}<0.5^{\circ}\right)$ in phase angle (Fig. 3). With added noise, the errors in $\mathrm{G}^{*}$ and $\delta$ increased to $\sim 2 \%\left(\mathrm{SD} 2-5 \%\right.$ ) and $\sim 1^{\circ}$ $\left(\mathrm{SD} \sim 2^{\circ}\right.$ ), respectively (Figs. 2 and 3 ). We did not find any significant dependence of the mean errors in magnitude and phase angle on the oscillatory frequency.

The remanent field in the cellular measurements was $B_{\mathrm{o}}=1.70 \pm 0.39 \mathrm{nT}$ (mean $\left.\pm \mathrm{SD}\right)$, which decayed with a time constant $\gamma=(2.4 \pm 1.5) \times 10^{-4} \mathrm{~s}^{-1}$. Fig. 4 shows the $\mathrm{G}^{*}(\omega)$ data obtained in the cells with frequency domain demodulation. The rotation amplitude of the beads was $\sim 5^{\circ}$. The storage modulus of the cells was approximately twice as large as the loss modulus. Both moduli increased linearly with frequency on a log-log plot with a similar slope $(\alpha \approx 1 / 4)$, which revealed a power law behavior with similar exponents. Nevertheless, G' had a slightly lower slope at high frequencies, and $G^{\prime \prime}$ had a lower slope at low frequencies. The loss tangent exhibited a nearly constant value of $\sim 0.5$ in the explored frequency range, although, in keeping with the slight frequency dependencies of the slopes observed in $\mathrm{G}^{\prime}$ and $\mathrm{G}^{\prime \prime}$, a slight parabolic tendency around $\sim 1 \mathrm{~Hz}$ can be appreciated. A linear regression analysis did not show any significant frequency dependence of $\mathrm{CV}$ of $\mathrm{G}^{\prime}$ and $\mathrm{G}^{\prime \prime}$. Assuming that intercellular variability does not depend on frequency, a constant $\mathrm{CV}$ indicates that the performance of the algorithm is similar at all frequencies.

The power law constant-phase model (Eq. 14) provided a good fit $\left(r^{2}=0.96\right)$ of the complex modulus of the cells (Fig. 4). The fitted parameters were $\mathrm{G}_{\mathrm{s}}^{\prime}=48.0$ $\mathrm{Pa}(\mathrm{SE}=1.95 \mathrm{~Pa})$ and $\alpha=0.27(\mathrm{SE}=0.01)$. The loss tangent of the model was $0.45(\tan \delta=\tan \pi \alpha / 2)$, which corresponds to a phase angle of $23^{\circ}$. Although the model provided a good overall description of the data, a slight underestimation of $\mathrm{G}^{\prime \prime}$ at low frequencies and overestimation $G^{\prime}$ at high frequencies can be observed (Fig. 4). Accordingly, the model fits the $\mathrm{G}^{\prime \prime} / \mathrm{G}^{\prime}$ data very well at $\sim 0.5 \mathrm{~Hz}$ and slightly underestimates this ratio at the ends of the explored frequency range.

\section{DISCUSSION}

Conventional methods of demodulation that have been employed previously in MTC confines measure- 
A

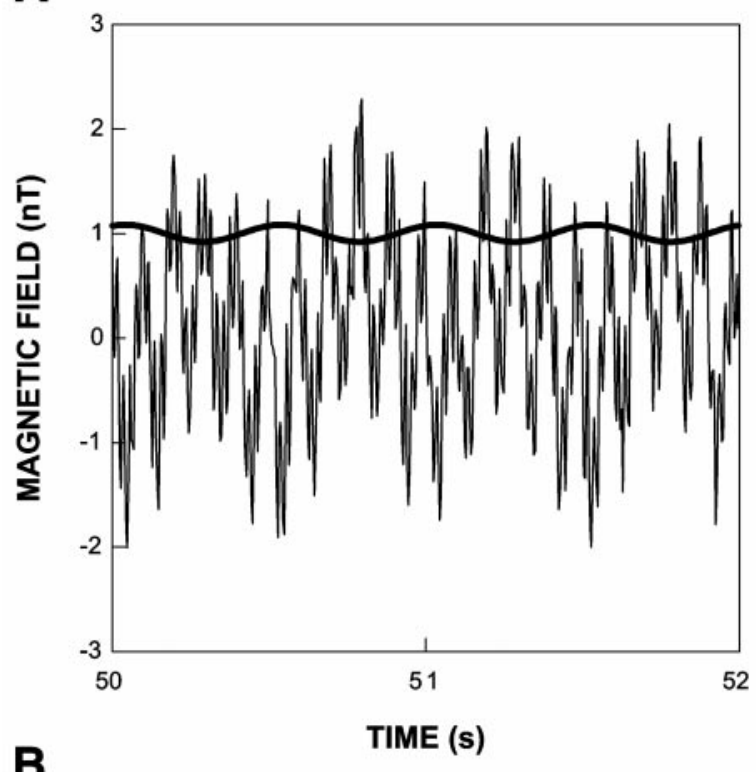

B

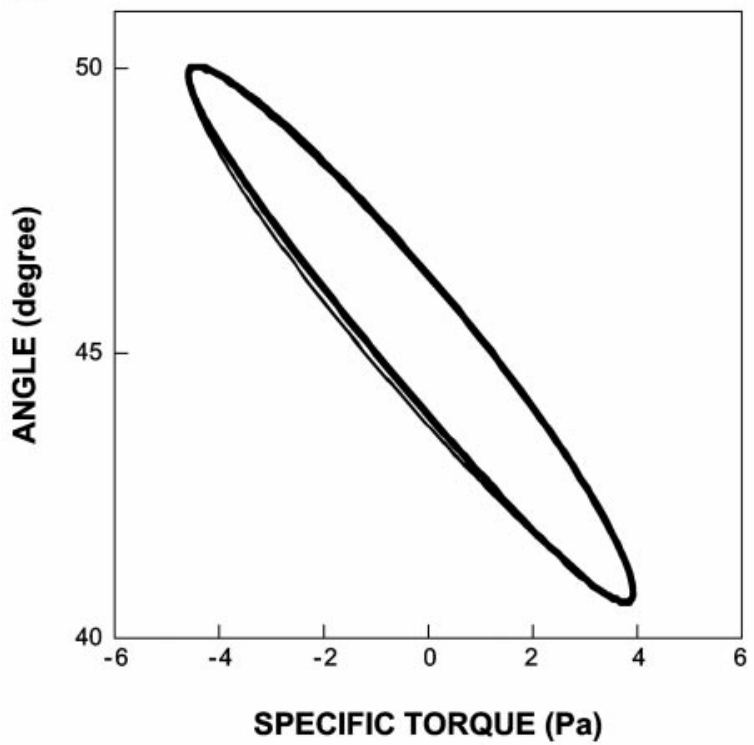

Fig. 2. Example of signal demodulation (2-s fragment) of a simulated $2-\mathrm{Hz}$ oscillatory recording. $A$ : magnetic field recorded by the magnetometer (thin line) and magnetic signal of the beads recovered with frequency domain demodulation (thick line). $B$ : specific torque vs. rotational strain loop computed from demodulation (thick line) compared with actual data (thin line).

ments to very low frequencies $(<0.5 \mathrm{~Hz})$. The new frequency domain demodulation algorithm developed in this work overcomes this limitation and dramatically extends oscillatory MTC measurements to higher frequencies. Using this new demodulation algorithm, we measured $\mathrm{G}^{*}$ in human bronchial epithelial cells over a frequency range spanning almost three decades $(0.03-16 \mathrm{~Hz})$. Both the storage and the loss moduli revealed a weak power law increase with frequency with similar exponents $(\alpha \approx 1 / 4)$. The loss tangent was $\sim 0.5$ and varied little with frequency. These features of the data conformed well to structural damping behavior (8) and the constant-phase law $(9,10)$.
Recently, the complex modulus of elasticity of smooth muscle cells has been obtained from 0.05 to 0.4 $\mathrm{Hz}$ with MTC time domain demodulation by applying an oscillatory twisting field (16). The angle and stress signals were first recovered by using conventional demodulation and were subsequently Fourier transformed to compute $\mathrm{G}^{*}$ in accordance with $E q$. 4. This approach yields a model-independent estimation of $\mathrm{G}^{*}$, but time domain demodulation restricts the measurements to low frequencies. By contrast, the new frequency domain demodulation has no intrinsic frequency limitations. In addition, measurements can be extended to lower frequencies provided that the duration of the recording covers at least one period of oscillation. The highest accessibly frequency is only restricted by the dynamic response of the magnetom-
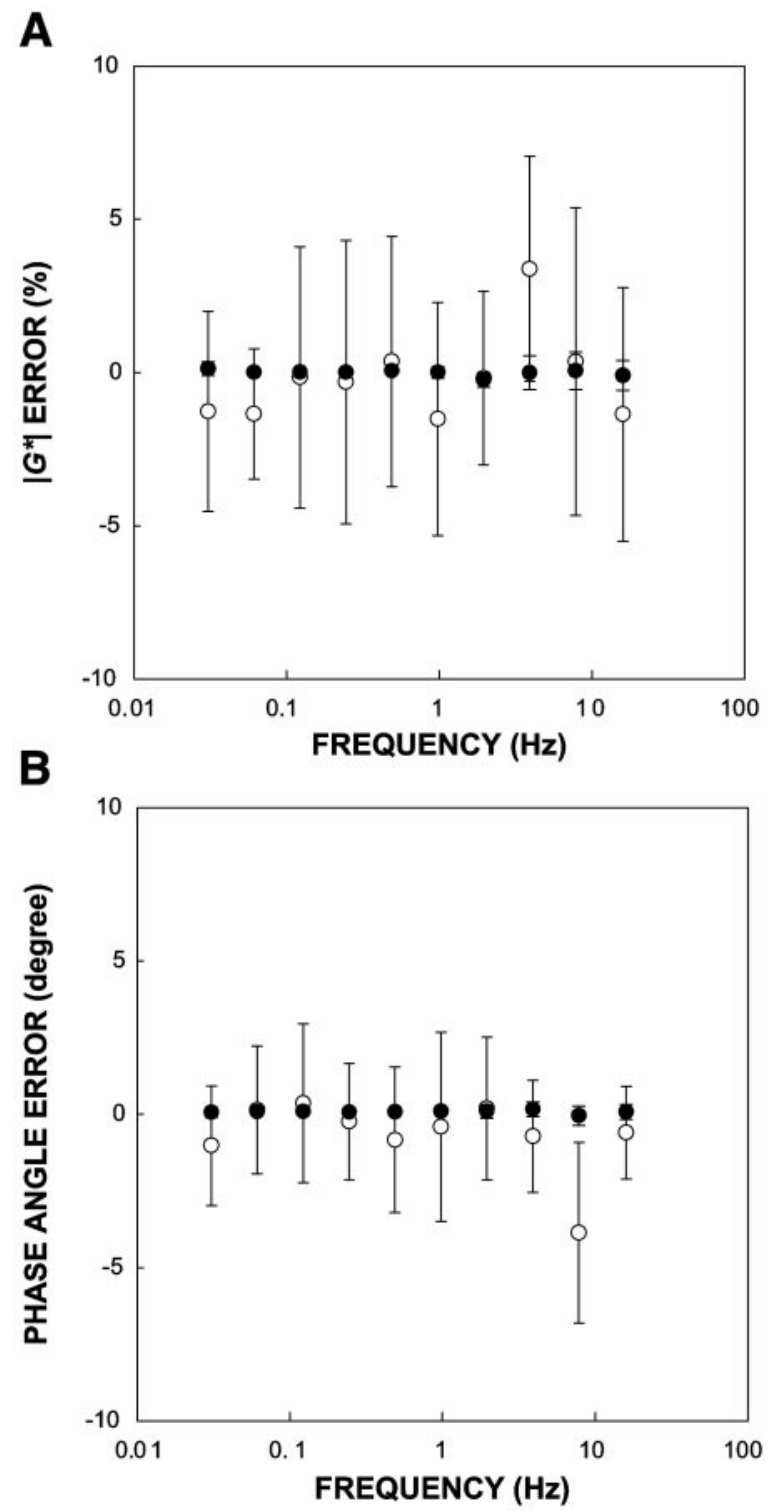

Fig. 3. Relative error in magnitude $(A)$ and absolute error in phase angle $(B)$ of the complex modulus of elasticity obtained with model simulation. $\bullet$ and $\circ$, Results obtained without and with random noise (root mean square $=0.17 \mathrm{nT})$, respectively. Data are means $\pm \mathrm{SE}$. 


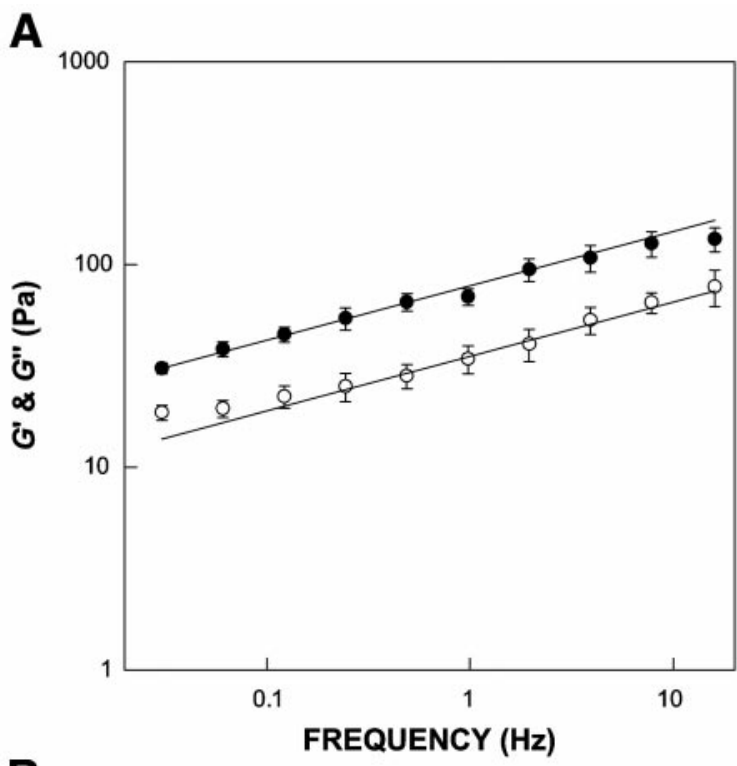

B

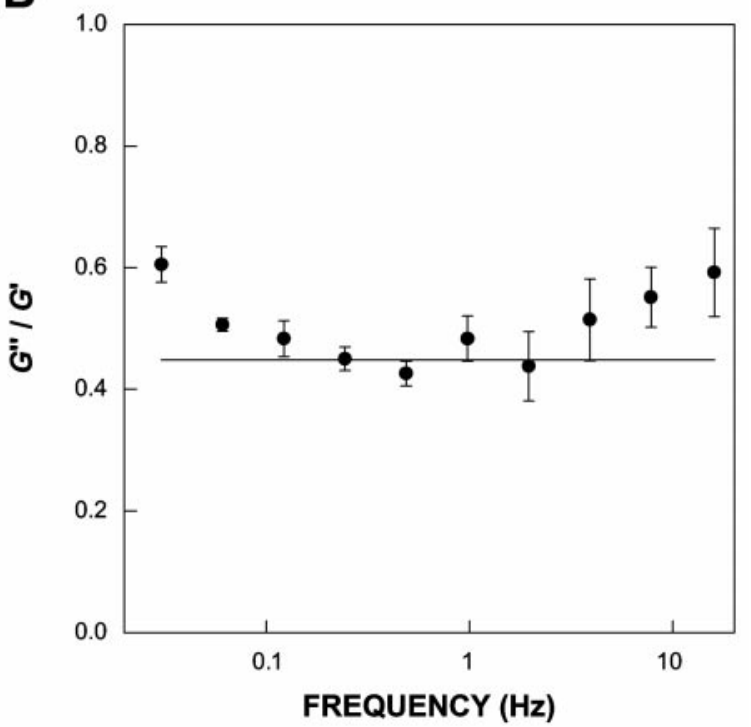

Fig. 4. Storage $\left(\mathrm{G}^{\prime}, \bullet\right)$ and loss $\left(\mathrm{G}^{\prime \prime}, \circ\right)$ moduli $(A)$ and loss tangent $\left(\mathrm{G}^{\prime \prime} / \mathrm{G}^{\prime}\right)(B)$ measured in cultured human bronchial epithelial cells. Solid lines are a fit of the power law constant-phase model to the complex modulus $\mathrm{G}^{*}(\omega)=\mathrm{G}_{\mathrm{s}}^{\prime}(1+j \eta)\left(\omega / \omega_{\mathrm{s}}\right)^{\alpha}$ with $\eta=\tan (\pi \alpha / 2)$ and $\omega_{\mathrm{s}}=1 \mathrm{~s}^{-1}$. Best-fit parameters are $\mathrm{G}_{\mathrm{s}}^{\prime}=48.0 \mathrm{~Pa}$ and $\alpha=0.27$.

eter. With the fluxgate magnetometer employed in this work (cutoff $200 \mathrm{~Hz}$ ), the highest accessible frequency is $20-30 \mathrm{~Hz}$. Novel solid-state magnetoresistive sensors with bandwidth on the order of megahertz may dramatically extend the frequency range of MTC measurements. It is noteworthy that the new algorithm can be also used to track changes in cell mechanical properties by demodulating the recordings using a moving time window.

There are a number of critical points in the new demodulation algorithm. First, oscillation frequency, sampling frequency, and experiment duration are adjusted to compute FFT of a whole number of periods of oscillation. This minimizes windowing leakage, and the oscillation components appear as narrow peaks in single spectral bins at $k \omega_{\mathrm{o}}(k=0,1, \ldots)$. Moreover, this maximizes SNR because only the noise located within these single bins are added to the signal. Second, oscillation and spinning frequencies must also be adjusted to prevent that their sum or difference is very close to $k \omega_{0}(k=0,1, \ldots)$. Third, $E q$. 10 assumes that the reference and the carrier are identical. The reference is generated cycle by cycle as a sinus synchronized with each revolution of the sample. However, possible misalignment of the sample or the magnetic probes could result in cyclic distortion of the modulation carrier, which would reduce the accuracy of spectral peak estimation. The demodulation algorithm cannot be tested in cellular experiments given that it is not possible to know the actual magnetic signal generated by the beads, $B(t)$. Therefore, we assessed the performance of the algorithm with a realistic simulation in a viscoelastic mathematical model. This simulation mimicked the relevant features of actual measurements allowing us to analyze the effect of each factor separately.

The high accuracy in the $G^{*}$ and $\delta$ estimates from the simulation model obtained without noise demonstrates that the algorithm corrects very well for spinning instability, spill field, magnetic relaxation, and magnetic contamination of the sample holder. Consequently, error in the simulation with noise can be attributed almost exclusively to the random noise within the single bins located at the harmonics of the oscillation frequency. We thus achieved the optimal SNR accessible from the recordings. With the usual values of $B_{\mathrm{o}}$ obtained in cellular measurements $(\sim 1$ $\mathrm{nT})$, the amplitude of the oscillatory response $(\sim 0.1 \mathrm{nT})$ is comparable to the level of random noise. Given that most of the power of the noise is concentrated at low frequencies $(<0.1 \mathrm{~Hz})$ and that multiplication by the reference shifts the noise spectrum to $\omega_{c}$, oscillatory measurements with frequency close to $\omega_{c}$ should be avoided. Moreover, the error can be made arbitrarily small by increasing the duration of the recording, which narrows the width of the frequency bins $(\Delta f=$ 1 /duration). Therefore, the results obtained in this simulation demonstrate an excellent performance of the algorithm and indicate that its accuracy does not depend on frequency.

The complex elastic modulus of the bronchial epithelial cells revealed a weak power law dependence on frequency dominated by elastic stresses. The rise of $\mathrm{G}^{*}$ with frequency indicates that the ability of the cells to resist deformation increases with the rate of deformation. However, the proportion of elastic vs. frictional stress varied little with frequency. The power law constant-phase model (Eq. 14) described the oscillatory mechanics of the cells very well. Consequently, only two independent parameters were needed to characterize the viscoelastic moduli of the cells in the explored frequency range. One parameter, $\mathrm{G}_{\mathrm{s}}^{\prime}$, scales the magnitude of both $G^{\prime}$ and $G^{\prime \prime}$ and provides an index of cell rigidity at $\omega=\omega_{\mathrm{s}}$. The other parameter, $\alpha$, accounts for the frequency dependence of both moduli and for their relative proportion. This is in agreement with the 
Kramers-Kronig relationships, which state that one of the two moduli can be estimated from the frequency dependence of the other one $(3,7)$. In particular, $\mathrm{G}^{\prime \prime}$ can be approximated as $1 / 2 \pi \mathrm{dG}^{\prime} / \mathrm{d} \ln \omega$ (3). Therefore, the storage and loss moduli appear to be interrelated and their power law frequency dependence implies a constant tangent ratio between them. From this interpretation, it follows that the degree of solid- or liquidlike character of the cells is associated with the slope of the moduli $\left[\mathrm{G}^{\prime \prime} / \mathrm{G}^{\prime}=\tan (\pi \alpha / 2)\right]$. A nearly constant loss tangent has been observed in many biological tissues, suggesting a coupling of elastic and dissipative processes at the level of the stress-bearing elements (structural damping law) (8).

Transforming $E q$. 14 to the time domain shows that the stress relaxation function $[G(t)]$ decreases with time as $\sim t^{-\alpha}$ (10). Furthermore, the relaxation spectrum $H(\tau)$ can be approximated as $-\mathrm{dG} / \mathrm{d} \ln t(7)$. Thus the viscoelastic behavior of the cells can be described with a continuous distribution of internal relaxation time constants whose contribution decreases as power law $H(\tau) \sim \tau^{-\alpha}$. A power law spectrum indicates that the internal relaxation processes exhibited no intrinsic time scale. The constant-phase model captured the main features of the rheological behavior of the cells over a wide frequency range around physiological frequencies. Nevertheless, one should not extrapolate this behavior too far to the extremes of frequency. Both $\mathrm{G}^{\prime}$ and $\mathrm{G}^{\prime \prime}$ of the model tend to zero when frequency approaches zero, and they are not bounded when frequency tends to infinity (Eq. 14).

The apparent viscosity of the cell has been computed in MTC step experiments by analyzing the stress recovery after the twisting step in terms of a parallel arrangement of a spring and a dashpot (Voigt body) $(20,24)$. This simple model has an exponential step response, whose time constant $(\tau)$ allows the computation of the apparent viscosity of the cell. This model interpretation predicts a liquidlike regime at low frequencies $(\omega \approx 1 / \tau)$ and a solidlike regime at high frequencies. This biphasic behavior does not agree with the weak positive frequency dependence of $G^{\prime}$ and $G^{\prime \prime}$ with $\mathrm{G}^{\prime \prime} / \mathrm{G}^{\prime} \approx 0.5$ that we found over approximately three frequency decades. Furthermore, in contrast to the constant viscosity assumed in the Voigt body, we found a power law negative frequency dependence of dynamic viscosity $\left(G^{\prime \prime} / \omega \sim \omega^{-3 / 4}\right)$. These differences indicate that a Voigt body is too simple a model to characterize the rheological behavior of the cell.

Model-free measurements of the complex elastic modulus at low frequencies $(0.05-0.4)$ have recently been obtained in cultured human airway smooth muscle cells with conventional MTC demodulation (16). These data compare reasonably well with our findings obtained over a wider frequency band in bronchial epithelial cells. Indeed, under baseline conditions, the airway smooth muscle cells showed an elastic modulus with a magnitude and power law frequency dependence $\left(G^{\prime}=57.3 \omega^{0.2} \mathrm{~Pa}\right)(16)$ similar to our data. The oscillatory response was also dominated by elastic stresses with a more solidlike behavior $\left(\mathrm{G}^{\prime \prime} / \mathrm{G}^{\prime} \approx 0.35\right)$.
However, the loss modulus of the airway smooth muscle cells did not show frequency dependence. This discrepancy with the weak power law dependence of $G^{\prime \prime}$ we found in the bronchial epithelial cells may be due to the limited frequency range of the previous study (approximately one decade). Thus these two cell types seem to have similar baseline rheological behavior.

Magnetic particles have recently been used to measure viscoelastic properties of the cell by tracking the displacement of single particles with digital video microscopy (time resolution of $0.04 \mathrm{~s}$ ) in creep experiments (2). Force steps of $\sim 1 \mathrm{~s}$ duration were applied to fibronectin-coated paramagnetic beads $(4.5 \mu \mathrm{m})$ attached to the membrane of adhering fibroblasts. The creep response was described as an initial elastic deformation followed by a viscoelastic relaxation, with $\tau$ $\sim 0.1 \mathrm{~s}$, and a final viscous regime with a constant deformation rate. This response was interpreted as a mechanical model consisting of a dashpot arranged in series with a standard linear solid (Voigt body in parallel with a spring). This model has three independent parameters featuring liquid- and solidlike regimes at low and high frequencies, respectively, with a viscoelastic regime transition at $f \sim 1 \mathrm{~Hz}(\omega=1 / \tau)$. The same three-phasic behavior $(\tau \sim 0.2 \mathrm{~s})$ was described when the creep experiments were performed with magnetic beads internalized by phagocytosis (1). The oscillatory behavior inferred from the model does not agree with our data obtained from direct measurements. Instead of a three-phasic regime, we found a weak power law rise in $\mathrm{G}^{*}$ with little variation in the loss tangent over approximately three frequency decades. This discrepancy could be due to the limited time resolution of the video recordings and to the use of a model with a single time constant to describe the data. In agreement with this explanation, a power law increase in the complex modulus and a loss tangent $(\sim \pi / 4)$ varying little over five frequency decades $\left(\sim 10^{-1}-10^{4} \mathrm{~Hz}\right)$ has been reported by laser tracking the Brownian motion of endogenous granules in kidney epithelial cells (27). In keeping with our findings, this behavior may reflect physical processes exhibiting a continuous distribution of internal time constants falling with increasing relaxation times as a power law $\left(\sim \tau^{-\alpha}\right)$. This interpretation also agrees with the smooth positive frequency dependence of stiffness and the small variation of the loss tangent $(\sim 0.5)$ reported $(0.2-200 \mathrm{~Hz})$ in cultured rat atrial myocytes with atomic force microscopy (18).

In conclusion, the new frequency domain demodulation algorithm developed in this work overcomes the frequency limitations of conventional MTC measurements. This improvement allows the measurement of oscillatory mechanics of the cell over a wide frequency range restricted only by the dynamic response of the magnetic sensor. Using this new approach, we measured the complex modulus of elasticity of cultured human airway epithelial cells from 0.03 to $16 \mathrm{~Hz}$. The cells revealed a predominantly elastic behavior with a constant proportion of elastic and friction stresses. $\mathrm{G}^{\prime}$ approximately doubled elastic modulus, and both in- 
creased with frequency as a power law $\left(\sim \omega^{\alpha}\right)$ with a weak exponent $(\alpha \approx 1 / 4)$. This reflects a continuous distribution of internal relaxation time constants falling with increasing relaxation times as a power law $\left(\sim \tau^{-\alpha}\right)$. The viscoelastic behavior observed in these cells can be described with only two independent parameters. One parameter provides an index of cell rigidity and scales the magnitude of $\mathrm{G}^{\prime}$ and $\mathrm{G}^{\prime \prime}$, and the other parameter accounts for the frequency dependence of both moduli and for their relative proportion. This mechanical behavior conforms to the structural damping law.

We thank Miguel Rodriguez for assistance in the experimental protocol and Ramón Farré, Mar Rotger, James P. Butler, and Ben Fabry for helpful comments and suggestions.

This study was supported by Grants DGESIC-PM980027, CICYTSAF990001, NIH-P01-HL-33009, NIH-R01-HL-65960, and the Whitaker Foundation.

\section{REFERENCES}

1. Bausch AR, Möller W, and Sackmann E. Measurement of local viscoelasticity and forces in living cells by magnetic tweezers. Biophys J 76: 573-579, 1999.

2. Bausch AR, Ziemann F, Boulbitch AA, Jacobson $\mathbf{K}$, and Sackmann E. Local measurements of viscoelastic parameters of adherent cell surfaces by magnetic bead microrheometry. Biophys J 75: 2038-2049, 1998.

3. Booij HC and Thoone GPJM. Generalization of KramersKronig transforms and some approximations of relations between viscoelastic quantities. Rheol Acta 21: 15-24, 1982

4. Crick FHC. The physical properties of cytoplasm. A study by means of the magnetic particle method. Part 2. Theoretical treatment. Exp Cell Res 1: 505-533, 1950.

5. Crick FHC and Hughes AFW. The physical properties of the cytoplasm. A study by means of the magnetic particle method. Part 1. Exp Cell Res 1: 37-80, 1950.

6. Duszyk M, Schwab B III, Zahalak GI, Quian H, and Elson EL. Cell poking: quantitative analysis of indentation of thick viscoelastic layers. Biophys J 55: 683-690, 1989.

7. Ferry JD. Viscoelastic Properties of Polymers. New York: Wiley, 1980.

8. Fredberg JJ and Stamenovic D. On the imperfect elasticity of lung tissue. J Appl Physiol 67: 2408-2419, 1989.

9. Hantos Z, Daróczy B, Suki B, Nagy S, and Fredberg JJ. Input impedance and peripheral inhomogeneity of dog lungs. J Appl Physiol 72: 168-178, 1992.

10. Hildebrandt J. Comparison of mathematical models for cat lung and viscoelastic balloon derived by Laplace transform methods from pressure-volume data. Bull Math Biophys 31: 651-667, 1969

11. Hubmayr RD, Shore SS, Fredberg JJ, Planus E, Panettieri RA Jr, Moller W, Heyder J, and Wang N. Pharmacological activation changes stiffness of cultured human airway smooth muscle cells. Am J Physiol Cell Physiol 271: C1660-C1668, 1996.

12. Ingber $\mathbf{D}$, Heidemann SR, Lamoureux $\mathbf{P}$, and Buxbaum RE. Opposing views on tensegrity as a structural framework for understanding cell mechanics. J Appl Physiol 89: 1663-1678, 2000.

13. Ingber DE, Prusty D, Sun Z, Betensky H, and Wang N. Cell shape, cytoskeletal mechanics, and cell cycle control in angiogenesis. J Biomech 28: 1471-1484, 1995.

14. Janmey PA. The cytoskeleton and cell signaling: component localization and mechanical coupling. Physiol Rev 78: 763-781, 1998.

15. Laporte JD, Moore PE, Abraham JH, Maksym GN, Fabry B, Panettieri RA Jr, and Shore SA. Role of ERK MAP kinases in response of cultured human airway smooth muscle cells to IL-1ß. Am J Physiol Lung Cell Mol Physiol 277: L943-L951, 1999.

16. Maksym GN, Fabry B, Butler JP, Navajas D, Tschumperlin DJ, Laporte JD, and Fredberg JJ. Mechanical properties of cultured human airway smooth muscle cells from 0.05 to 04 Hz. J Appl Physiol 89: 1619-1632, 2000.

17. Marmarelis PZ and Marmarelis VZ. Analysis of Physiological Systems: The White Noise Approach. New York: Plenum, 1978.

18. Schroff SG, Saner DR, and Lal R. Dynamic micromechanical properties of cultured rat atrial myocytes measured by atomic force microscopy. Am J Physiol Cell Physiol 269: C286-C292, 1995

19. Stamenovic D and Wang N. Engineering approaches to cytoskeletal mechanics. J Appl Physiol 89: 2085-2090, 2000.

20. Tagawa H, Wang N, Narishige T, Ingber DE, Zile MR, and Cooper G IV. Cytoskeletal mechanics in pressure-overload cardiac hypertrophy. Circ Res 80: 281-289, 1997.

21. Valberg PA. Magnetometry of ingested particles in pulmonary macrophages. Science 224: 513-516, 1984.

22. Valberg PA and Butler JP. Magnetic particle motions within living cells. Physical theory and techniques. Biophys J 52: 537550,1987

23. Wang N, Butler JP, and Ingber DE. Mechanotransduction across the cell surface and through the cytoskeleton. Science 260: 1124-1127, 1993.

24. Wang $\mathbf{N}$ and Ingber DE. Control of cytoskeletal mechanics by extracellular matrix, cell shape, and mechanical tension. Biophys J 66: 2181-2189, 1994.

25. Wang $\mathbf{N}$ and Ingber DE. Probing transmembrane mechanical coupling and cytomechanics using magnetic twisting cytometry. Biochem Cell Biol 73: 327-335, 1995.

26. Wang N, Planus E, Pouchelet M, Fredberg JJ, and Barlovatz-Meimon G. Urokinase receptor mediates mechanical transfer across the cell surface. Am J Physiol Cell Physiol 268: C1062-C1066, 1995.

27. Yamada S, Wirtz D, and Kuo SC. Mechanics of living cells measured by laser tracking microrheology. Biophys $J$ 78: 1736$1747,2000$.

28. Yanai M, Butler JP, Suzuki T, Kanda A, Kurachi M, Tashiro H, and Sasaki H. Intracellular elasticity and viscosity in the body, leading, and trailing regions of locomoting neutrophils. Am J Physiol Cell Physiol 277: C432-C440, 1999. 